\section{Estratégia de recrutamento de fumantes no metrô do Rio de Janeiro, Brasil, para ampliar o acesso a linhas telefônicas de apoio à cessação: impacto da novidade}

\author{
Recruitment of smokers in the Rio de Janeiro \\ subway, Brazil, as a strategy to increase access \\ to quitline services: the impact of novelty
}

\author{
André Salem Szklo ${ }^{1}$ \\ Evandro da Silva Freire Coutinho ${ }^{2}$ \\ Helena Maria Tannhauser Barros ${ }^{3}$ \\ Cristina Perez ${ }^{1}$ \\ Taís de Campos Moreira 3 \\ Luciana Rizzieri Figueiró 3 \\ Mariana Pinho 1 \\ Valeska Figueiredo Carvalho ${ }^{1}$
}

\begin{abstract}
1 Coordenação de Prevenção e Vigilância, Instituto Nacional de Câncer, Rio de Janeiro,

Brasil.

2 Escola Nacional de Saúde Pública Sergio Arouca, Fundação Oswaldo Cruz, Rio de Janeiro, Brasil.

3 Departamento de

Ciências Básicas da Saúde, Universidade Federal de Ciências da Saúde de Porto Alegre, Porto Alegre, Brasil.
\end{abstract}

Correspondência A. S. Szklo

Coordenação de Prevenção e Vigilância, Instituto Nacional de Câncer.

Rua dos Inválidos 212,

Rio de Janeiro, $R J$

20231-020, Brasil.

aszklo@inca.gov.br

\section{Abstract}

Creative and innovative strategies to recruit smokers are essential for improving tobacco control activities. Currently in Brazil, through health warning messages on cigarette packs, there is a permanent and intense spread of messages that provoke feelings of loss associated with smoking, which is important to encourage access to smoking quitlines. The study analyzed the call rate for telephone counseling after introducing a new strategy for reactive recruitment focused on the theme "smoking causes shortness of breath", adapted to the subway setting in Rio de Janeiro, as compared to the rates for two existing reactive strategies. Regardless of age bracket, there was a higher response to the new proposed strategy. Despite the major awareness-raising in Brazil concerning the ills of tobacco, new communications formats approaching personally relevant themes can increase the number and range of smokers recruited for telephone counseling to support cessation.

Tobacco Control Campaigns; Tobacco Use Cessation; Smoking

\section{Introdução}

Apesar de pesquisas recentes apontarem para um declínio na prevalência de fumantes no Brasil 1,2, existe um terreno ainda fértil para aprimorar as ações de estímulo à cessação do tabagismo no país. Tais iniciativas representam um compromisso de saúde pública que vai ao encontro do artigo 14 do tratado internacional para controlar o tabagismo no mundo ( $W H O$ Framework Convention on Tobacco Control FCTC) ${ }^{3}$, do qual o Brasil é signatário.

O resultado dessas ações é função da eficácia do tipo de intervenção proposta e dos instrumentos de recrutamento de fumantes elegíveis utilizados 4 . Esses instrumentos podem envolver canais de comunicação sem contato pessoal direto com o pesquisador ou o provedor do serviço (canais reativos), os quais conseguem atingir uma população elegível mais numerosa a um menor custo 4,5 . A literatura aponta, contudo, para uma taxa de resposta muito baixa ao recrutamento reativo, variando entre $0,1 \%$ e $7 \% 4$. Dessa forma, a novidade, impulsionada pela criatividade do veículo escolhido para divulgar temas originais e relevantes ao público elegível, é vista como um elemento essencial para aumentar a resposta desejada 5,6.

Atualmente, no Brasil, por meio das imagens de advertência presentes nos maços de cigarro, há uma divulgação permanente e intensa de mensagens que provocam sentimentos de perda 
associados ao tabagismo. Junto às advertências, encontra-se o número telefônico do Serviço Disque Saúde/Pare de Fumar (DPF) 7, que se constitui em um serviço gratuito do Ministério da Saúde para orientação de fumantes sobre como parar de fumar. Outra linha telefônica também gratuita, Serviço Nacional de Orientações e Informações sobre a Prevenção do Uso Indevido de Drogas - VIVAVOZ (Secretaria Nacional de Políticas sobre Drogas/Universidade Federal de Ciências de Saúde de Porto Alegre; http://psico ativas.ufcspa.edu.br/vivavoz, acessado em 09/ Jun/2009), é divulgada ininterruptamente através de um texto neutro-explicativo presente na Internet com o objetivo de tratar usuários de drogas, incluindo fumantes.

Neste estudo, comparou-se a taxa de ligação observada após a introdução de uma nova estratégia de recrutamento reativo para ampliar o acesso à intervenção "aconselhamento telefônico de apoio à cessação de fumar”, com as taxas verificadas, no mesmo período, para duas outras estratégias reativas existentes. Para as considerações mencionadas aqui, não existem, até o momento, registros em literatura indexada.

\section{Metodologia}

As três estratégias de recrutamento reativo para aconselhamento telefônico de fumantes comparadas neste estudo foram:

Estratégia 1: maços de cigarros contendo mensagens com conteúdo "negativo" utilizados, desde 2002, como principal veículo de divulgação do serviço DPF. O serviço é oferecido através de atendimento humano de segunda à sextafeira, das 07h às 19h, exceto feriados. Ademais, conta com mensagens gravadas 24 horas por dia, sete dias por semana;

Estratégia 2: página de conteúdo neutro na Internet utilizada, desde 2005, como principal veículo de divulgação do Serviço VIVAVOZ. O serviço funciona das $08 \mathrm{~h}$ às $24 \mathrm{~h}$, de segunda à sexta-feira, exceto feriados;

Estratégia 3: Projeto-Metrô para divulgação do serviço VIVAVOZ ("serviço PM-VIVAVOZ"), descrito a seguir.

Conduziu-se, durante todo o mês de abril de 2008, um estudo de intervenção em estações selecionadas do metrô do Município do Rio de Janeiro. Para encorajar os fumantes a ligarem para o serviço VIVAVOZ, foram definidas duas abordagens contendo mensagens com conceitos distintos - ganhos ao parar de fumar versus perdas ao continuar fumando -, as quais foram expostas em estações diferentes. A fim de garantir a comparabilidade dos conceitos subjacentes às estra- tégias de recrutamento que se queria comparar, este artigo foi focado apenas nos resultados do estudo de intervenção relacionados ao grupo que recebeu o estímulo de conteúdo "negativo".

Cartazes desenvolvidos por profissionais de Comunicação, Marketing \& Design abordando o tema específico da perda/falta de fôlego foram expostos em lugares estrategicamente posicionados das plataformas de embarque da estação - pilastras divisórias centrais - diariamente das 05h às 24 h (Figura 1). Em cada cartaz constava também o telefone do serviço VIVAVOZ. As ligações eram associadas à nova estratégia de recrutamento de fumantes quando se respondia "cartaz no metrô" à seguinte pergunta: "Como o(a) Sr(a) soube desse número?".

\section{Análise de dados}

Foram calculadas as taxas de ligação gerais e específicas por idade para os serviços de aconselhamento telefônico citados anteriormente, com intuito de avaliar o impacto das estratégias de recrutamento vinculadas a eles.

Como o denominador das taxas (população de fumantes) não era conhecido, ele foi estimado pela aplicação das prevalências de tabagismo corrigidas, específicas por sexo e faixa etária, aos usuários da estação de metrô selecionada (base do "serviço PM-VIVAVOZ"), à população geral do Município do Rio de Janeiro (base do serviço DPF) e à respectiva população usuária de Internet (base do serviço VIVAVOZ). Essas prevalências são oriundas do inquérito domiciliar conduzido no Município do Rio de Janeiro, em 2003, e foram corrigidas para o ano de 2007, extrapolando-se a tendência de queda linear observada nas prevalências específicas entre 1989 (ano de condução da Pesquisa Nacional sobre Saúde e Nutrição) e 20032.

Em seguida, procedeu-se a um ajuste pelo método direto ${ }^{8}$ para estimar as novas taxas gerais de ligação dos serviços analisados, tendo como padrão a população de usuários fumantes da estação de metrô selecionada.

Finalmente, as razões das taxas de ligação e os respectivos intervalos de $95 \%$ de confiança, específicas e geral, para o PM-VIVAVOZ comparado com DPF e VIVAVOZ, foram calculadas pelo programa estatístico PEPI 4.0 (Computer Programs for Epidemiologists; http://www.sagebrush press.com/pepi). Foi realizado ainda um teste de tendência linear para as razões de taxas de ligação específicas por idade encontradas. 


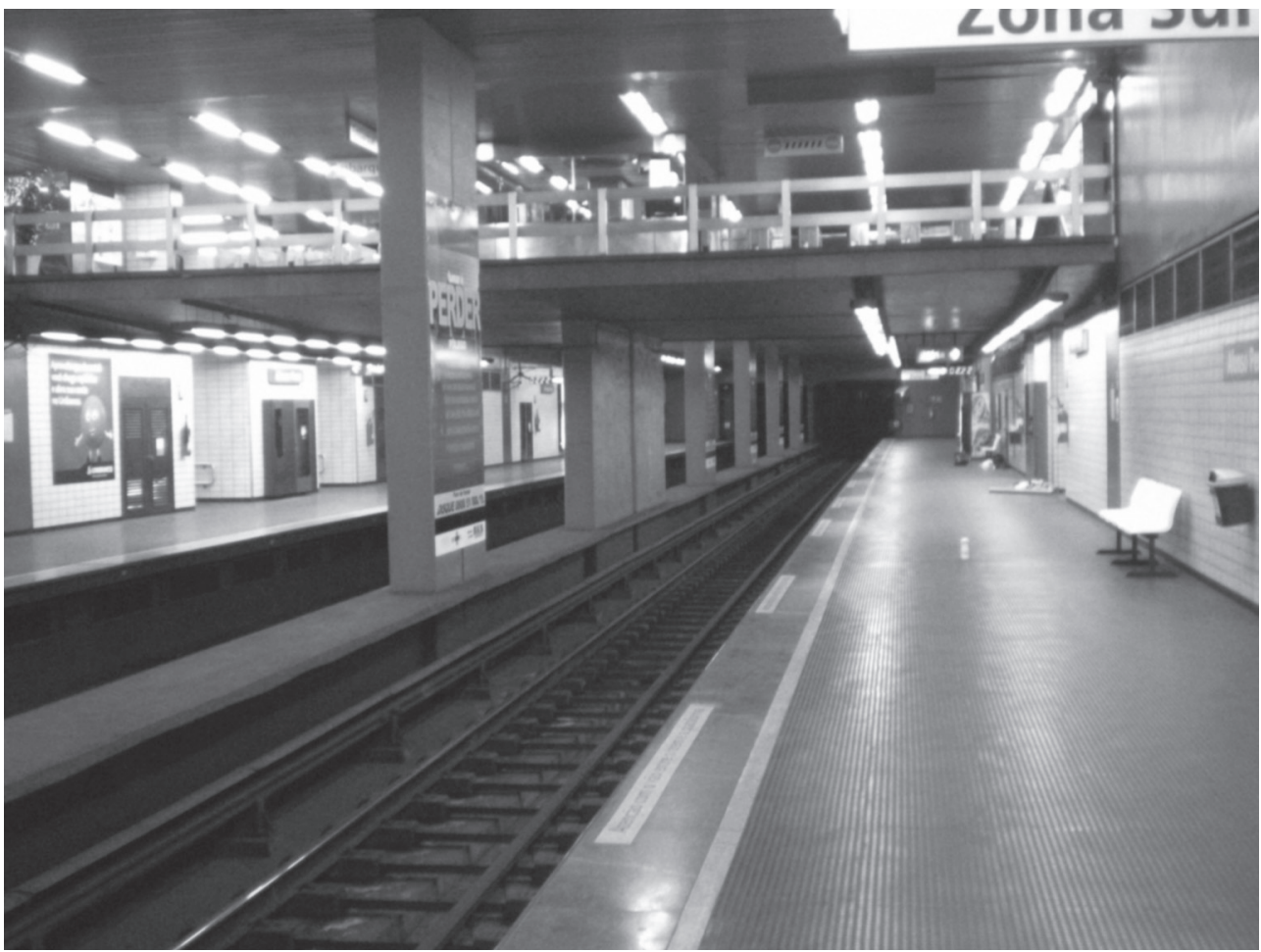

\section{Resultados}

A taxa de ligação estimada para o PM-VIVAVOZ foi cerca de 4 e 700 vezes maior que aquelas estimadas, respectivamente, para o DPF e VIVAVOZ (Tabela 1). Após ajuste pela faixa etária, a razão nas taxas geral aumentou aproximadamente $20 \%$, na comparação com o DPF, e passou a ser aproximadamente 500 vezes maior (redução de 25\%) na comparação com o serviço VIVAVOZ.

As taxas do PM-VIVAVOZ por faixa etária foram sempre superiores às dos outros serviços, com exceção da faixa etária 15-24 anos do DPF. Verificou-se, ainda, na comparação PM-VIVAVOZ e DPF, uma tendência de aumento linear na razão de taxas de ligação com o avançar da idade $(\mathrm{p}<0,001)$.

\section{Conclusões}

O estudo sugere que, mesmo diante de um contexto de exposição massiva a informações sobre os malefícios do cigarro, inserido em um programa de ações abrangentes de controle do tabaco 9, uma estratégia inovadora pode ampliar consideravelmente o recrutamento para uso de linhas telefônicas de aconselhamento do fumante.

Mensagens negativas utilizadas durante longo tempo ficam super expostas, desgastam-se e, conseqüentemente, perdem impacto $4,5,6$. No caso do DPF, as mensagens existentes foram introduzidas há cinco anos. Ao efeito cumulativo no tempo do desgaste do conteúdo da mensagem, pode ser, provavelmente, adicionado o desgaste do próprio maço de cigarros como veículo para divulgação dos malefícios do tabagismo, dado que isso já ocorre, no Brasil, desde 1988 9. É possível que a escolha de um novo tema de relevância pessoal para jovens e idosos, homens e mulheres, tal como falta de fôlego, exposto nas pilastras di- 
Distribuição das taxas de ligação brutas * e ajustadas *,**, razões das taxas de ligações brutas e ajustadas, em função dos serviços utilizados, segundo faixa etária ***. Município do Rio de Janeiro, Brasil, 31 de março de 2008 a 30 de abril de 2008.

\begin{tabular}{|c|c|c|c|c|c|}
\hline \multirow[t]{2}{*}{ Idade (anos) } & \multicolumn{3}{|c|}{ Serviço utilizado (taxas de ligação) } & \multicolumn{2}{|c|}{ Serviços comparados (razões das taxas de ligações) } \\
\hline & DPF \# & VIVAVOZ \#\# & PM-VIVAVOZ \#\#\# & PM-VIVAVOZ vs. DPF § & PM-VIVAVOZ vs. VIVAVOZ \\
\hline $15-24$ & 290 & 0,0 & 270 & $0,9(0,3-2,5)$ & $-\S \S$ \\
\hline $25-34$ & 80 & 0,6 & 250 & $3,1(1,5-6,4)$ & $416,7(136,5-1.355,5)$ \\
\hline $35-44$ & 30 & 0,2 & 210 & $7,0(3,9-12,4)$ & $1.050,0(434,5-8.673,0)$ \\
\hline $45-54$ & 20 & 0,2 & 200 & $10,0(5,8-17,5)$ & $1.000,0(146,4-8.553,2)$ \\
\hline $55+$ & 20 & 0,4 & 250 & $12,5(6,7-22,4)$ & $625,0(79,5-4.786,6)$ \\
\hline Total bruto & 60 & 0,3 & 220 & $3,7(2,9-5,7)$ & $733,3(357,5-1.399,9)$ \\
\hline Total ajustado & 50 & 0,4 & NA & $4,4(3,4-6,0)$ & $550,0(294,0-1.004,2)$ \\
\hline
\end{tabular}

DPF: Serviço Disque Saúde/Pare de Fumar; VIVAVOZ: Serviço Nacional de Orientações e Informações sobre a Prevenção do Uso Indevido de Drogas; PMVIVAVOZ: Projeto-Metrô para divulgação do Serviço VIVAVOZ; NA: não se aplica.

* Taxas brutas e ajustadas expressas em 10 mil fumantes-mês;

** Ajuste direto pela população de usuários fumantes da estação de metrô selecionada. Distribuição etária: 15-24, 7,1\%; 25-34, 13,6\%; 35-44, 27,2\%; 45-54 $30,6 \% ; 55+, 21,3 \%$;

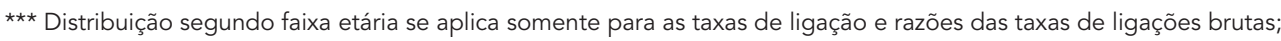

\# Veiculado principalmente nos maços de cigarros. População de fumantes estimada: 15-24, 69.124; 25-34, 135.210; 35-44, 231.316; 45-54, 214.116; 55+, 138.460. Número de ligações registradas: 7.098 , considerando em média $20 \%$ de ligações de não fumantes e $10 \%$ de ligações repetidas;

\#\# Veiculado primordialmente pela Internet. População de fumantes estimada para usuários de internet: 15-24, 55.299; 25-34, 87.886; 35-44, 92.527; 45-54, 55.429; 55+, 25.074. Número de ligações de fumantes registradas: 10;

\#\#\# Divulgado por cartazes em uma estação de metrô. População de fumantes estimada para a estação de metrô selecionada: 15-24, 150; 25-34, 286; 35-44,

573; 45-54, 644; 55+, 448. Número de ligações de fumantes registradas: 40;

$\S$ Teste de tendência linear: $\chi^{2}=36,02$ e $p<0,001$;

$\S \S$ Em função de não termos tido ligação neste estrato para o serviço VIVAVOZ, a razão de taxas deu um número infinito.

visórias centrais do metrô para conferir grande visibilidade e contato visual prolongado, tenha representado um dos principais fatores para o resultado observado. Em fumantes com idade mais avançada, este resultado parece ter sido ainda mais evidente, estando eles expostos ao "formato do maço de cigarros" há mais tempo.

Apesar de o serviço VIVAVOZ ter apresentado uma taxa de ligação muito baixa, o maior esforço dos fumantes em buscar informação não acessível de imediato (i.e., portal não específico para fumantes na rede), diferentemente do que ocorreu com DPF e PM-VIVAVOZ, pode ter acarretado uma maior adesão à intervenção proposta e aumentado o impacto do serviço ${ }^{10}$. Contudo, a elaboração de campanhas de marketing qualificadas para divulgação do VIVAVOZ na Internet, ou em outros veículos, deveria ser estimulada visando aumentar a sua contribuição no apoio à cessação do tabagismo.

A magnitude das razões de taxas ajustadas deve ser vista, entretanto, com cautela por dois motivos: (i) a baixa precisão das estimativas (intervalos de confiança amplos); e (ii) a dependência entre o ajuste direto utilizado e a população de referência empregada, em função das razões das taxas de ligação específicas por faixa etária serem diferentes 8 . Outra limitação seria o fato de as populações de fumantes dos denominadores terem sido estimadas com base apenas nas distribuições por sexo e faixa etária. Porém, estas variáveis são as que melhor definem a prevalência de fumantes em uma população 11. Finalmente, a potencial "contaminação" entre as estratégias de recrutamento comparadas neste estudo não é passível de quantificação. Por exemplo, se os fumantes-usuários da estação de metrô selecionada tivessem ligado para o DPF em decorrência do estímulo da nova estratégia de captação utilizada, o resultado na taxa de ligação do PMVIVAVOZ teria sido subestimado.

Em função dos resultados obtidos, estratégias de captação de fumantes para serviços de aconselhamento telefônico de apoio à cessação poderiam considerar a integração com áreas diversas de geração de conhecimento (i.e., comunicação, marketing e epidemiologia) para incluir canais de comunicação inovadores e criativos de sensibilização de fumantes em ambientes de grande circulação de indivíduos. 


\section{Resumo}

Criatividade e inovação das estratégias de recrutamento de fumantes são fundamentais para aprimorar as ações de controle do tabagismo. Atualmente, no Brasil, por meio das imagens de advertência presentes nos maços de cigarros, há uma divulgação permanente e intensa de mensagens que provocam sentimentos de perda associados ao tabagismo, os quais são importantes para estimular o acesso a linhas telefônicas de apoio à cessação. Comparou-se a taxa de ligação para aconselhamento telefônico observada após introdução de nova estratégia de recrutamento reativo focada no tema "fumar é perder fôlego" e adaptada ao cenário do metrô do Rio de Janeiro, com as taxas verificadas para duas outras estratégias reativas existentes. Independentemente da faixa etária, houve maior resposta para a nova estratégia proposta. Apesar da grande sensibilização, no Brasil, quanto aos malefícios do tabaco, novos formatos de comunicação abordando temas de relevância pessoal podem aumentar a quantidade e diversidade da população de fumantes recrutada para serviços de aconselhamento telefônico de suporte à cessação.

Campanhas para o Controle do Tabagismo; Abandono do Uso de Tabaco; Tabagismo

\section{Referências}

1. Monteiro CA, Cavalcante T, Moura EC, Claro RM, Szwarcwald CL. Population-based evidence of a strong decline in the prevalence of smokers in Brazil (1989-2003). Bull World Health Organ 2007; 85:527-34.

2. Figueiredo VC. Um panorama do tabagismo em 16 capitais brasileiras e Distrito Federal: tendências e heterogeneidades [Tese de Doutorado]. Rio de Janeiro: Instituto de Medicina Social, Universidade do Estado do Rio de Janeiro; 2007.

3. World Health Organization. WHO Framework Convention on Tobacco Control. http://www.who.int/ fctc/text_download/en/index.html (acessado em 09/Jun/2009).

4. Szklo AS. Review of strategies to recruit smokers: a population impact perspective. Cad Saúde Pública 2008; 4 Suppl 4:S621-34.

5. Anderson CM, Zhu SH. Tobacco quitlines: looking back and looking ahead. Tob Control 2007; 16 Suppl:i81-6.

6. Krugman DM, Fox RJ, Fisher PM. Do cigarette warnings warn? Understanding what will take to develop more effective warnings. J Health Commun 1999; 4:95-104.

\section{Colaboradores}

A. S. Szklo participou do planejamento da coleta de dados, da análise de dados, discussões e elaboração do texto final desta nota de pesquisa. E. S. F. Coutinho, H. M. T. Barros, T. C. Moreira e L. R. Figueiró participaram do planejamento da coleta de dados, das discussões e elaboração do texto final desta nota de pesquisa. C. Perez, M. Pinho e V. F. Carvalho participaram das discussões e elaboração do texto final desta nota de pesquisa.

\section{Agradecimentos}

Rejane Spitz e Nilton Gamba Jr., do Departamento de Artes \& Design da Pontifícia Universidade Católica do Rio de Janeiro, pelo projeto de design das intervenções gráficas do Projeto-Metrô. Profissionais do Departamento de Comunicação da Concessionária Metrô-Rio, pelo suporte prestado durante a realização do Projeto-Metrô. O Projeto-Metrô foi financiado através do Programa de Cooperação Instituto Nacional de Câncer-Fundação Oswaldo Cruz (INCA-Fiocruz; convênio no. 953/2006) e recursos do próprio INCA (10302122087580033021830/2008).
7. Departamento de Ouvidoria Geral do SUS. Apresentação de dados do serviço Disque Saúde. Opção: como parar de fumar. http://portal.saude.gov. $\mathrm{br} /$ portal/arquivos/pdf/relatorio_tematico_\%20 inca.pdf (acessado em 09/Jun/2009).

8. Szklo M, Nieto FJ. Epidemiology beyond the basics. Gaithersburg: Aspen Publishers; 2000.

9. Cavalcante T. Experiencia brasileña con políticas de control del tabaquismo. Salud Pública Méx 2004; 46:549-58.

10. West R, Gilsenan A, Coste F, Zhou X, Brouard R, Nonnemaker J, et al. The ATTEMPT cohort: a multi-national longitudinal study of predictors, patterns and consequences of smoking cessation; introduction and evaluation of internet recruitment and data collection methods. Addiction 2006; 101:1352-61.

11. Lopez AD, Collishaw NE, Piha T. A descriptive model of the cigarette epidemic in developed countries. Tob Control 1994; 3:242-7.

Recebido em 08/Jan/2009

Versão final reapresentada em 26/Jun/2009 Aprovado em 09/Set/2009 